\title{
A convergence analysis of the exponential Euler iteration for nonlinear ill-posed problems
}

\author{
Marlis Hochbruck ${ }^{1}$, Michael Hönig ${ }^{1}$ and \\ Alexander Ostermann ${ }^{2}$ \\ 1 Mathematisches Institut, Heinrich-Heine Universität Düsseldorf, D-40225 \\ Düsseldorf, Germany \\ 2 Institut für Mathematik, Universität Innsbruck, A-6020 Innsbruck, Austria \\ E-mail: marlis@am.uni-duesseldorf.de, hoenig@am.uni-duesseldorf.de, \\ alexander.ostermann@uibk.ac . at
}

\begin{abstract}
Asymptotic regularization is a well established tool for treating nonlinear ill-posed problems. For its numerical realization, an appropriate numerical method for solving differential equations is required. In this paper, we analyze an exponential Euler method for this purpose. The scheme requires a certain approximation to the Jacobian of the Showalter differential equation and gives the exact solution for linear problems. Under a suitable discrepancy principle, the method is shown to be convergent under the same assumptions that are needed for the continuous analysis. Our convergence analysis admits variable step sizes and moreover yields optimal convergence rates.

Keywords: nonlinear ill-posed problems, asymptotic regularization, exponential integrators, variable step sizes, convergence, optimal convergence rates
\end{abstract}

Submitted to: Inverse Problems

AMS classification scheme numbers: 65J20, 65L05

\section{Introduction}

In this paper we consider the problem

$$
F(x)=y,
$$

where $F: \mathcal{D}(F) \subset X \rightarrow Y$ is a nonlinear differentiable operator between the Hilbert spaces $X$ and $Y$, whose Fréchet derivative $F^{\prime}(u)$ is locally uniformly bounded. In the following, we always assume that (1) has a solution $x_{*} \in \mathcal{D}(F)$ but we do not assume that this solution is unique. We are interested in ill-posed problems, where the solution does not depend continuously on the data $y$. In order to solve the perturbed problem

$$
F(u)=y^{\delta},
$$

with perturbed data $y^{\delta} \approx y$ satisfying

$$
\left\|y^{\delta}-y\right\| \leq \delta
$$

regularization is indispensable. Throughout the paper, the norm in both Hilbert spaces $X$ and $Y$ is denoted by $\|\cdot\|$, the corresponding inner product by $\langle\cdot, \cdot\rangle$. 
Among several regularization techniques, one possible option is asymptotic regularization based on the Showalter differential equation [14]

$$
u^{\prime}(t)=F^{\prime}(u(t))^{*}\left(y^{\delta}-F(u(t))\right), \quad t \geq 0, \quad u(0)=x_{0} .
$$

It was shown in [15] that the exact solution of (4) yields an optimal order regularization scheme under suitable assumptions. The explicit Euler method applied to (4) leads to the well-known Landweber iteration analyzed in [5]. In [7] we proposed to use the exponential Euler scheme for solving (4) numerically and we presented numerical examples which showed that this scheme is competitive for certain applications.

In this paper, we will analyze the convergence properties of the exponential Euler regularization method. In particular, we will prove that, under the same assumptions as for the continuous analysis of Tautenhahn [15], the scheme converges in the limit $\delta \rightarrow 0$ with the optimal convergence rate. Our convergence analysis is performed for variable step sizes satisfying a suitable discrepancy principle.

The paper is organized as follows. In Section 2 we briefly recall the exponential Euler regularization scheme and introduce some notation to improve the readability of this paper. Section 3 is devoted to the convergence analysis. The analysis in this section is motivated by previous work by Hanke [3]. The optimal convergence rates are shown in Section 4. The sections about the convergence both start with a review of the corresponding continuous results from Tautenhahn [15], so that the reader can easily compare the assumptions and the results. Finally, we comment on generalizations of our analysis to other regularization methods.

\section{Exponential Euler regularization}

The exponential Euler method is the simplest variant of the class of exponential Rosenbrock integrators [8]. It is based on the variation-of-constants formula which allows to integrate the linear part of semilinear differential equations exactly. In [7] we proposed to use the following modification of the original exponential Euler scheme

$$
u_{n+1}=u_{n}+h_{n} \varphi\left(-h_{n} J\left(u_{n}\right)\right) F^{\prime}\left(u_{n}\right)^{*}\left(y^{\delta}-F\left(u_{n}\right)\right), \quad u_{0}=x_{0}
$$

for solving (4). Here, we denote

$$
J(u)=F^{\prime}(u)^{*} F^{\prime}(u) .
$$

Hence, $-J\left(u_{n}\right)$ is an approximation to the Jacobian of the right-hand side of (4). Moreover, $\varphi$ denotes the entire function

$$
\varphi(z)=\frac{\mathrm{e}^{z}-1}{z} .
$$

If $u_{n} \approx u\left(t_{n}\right)$, then this scheme gives an approximation $u_{n+1} \approx u\left(t_{n+1}\right)$, at time

$$
t_{n+1}=t_{n}+h_{n}, \quad t_{0}=0 .
$$

For the convergence of the scheme it will be essential to use appropriate time steps $h_{n}$. Details will be discussed in Section 3.3. The iteration is stopped as soon as the standard discrepancy principle

$$
\left\|\Delta F_{n_{*}}^{\delta}\right\| \leq \tau \delta<\left\|\Delta F_{n}^{\delta}\right\| \quad \text { for all } n<n_{*},
$$

is satisfied. Here $\tau>1$ is a parameter and

$$
\Delta F_{n}^{\delta}=y^{\delta}-F\left(u_{n}\right)
$$


denotes the residual of the perturbed problem. The stopping criterion (8) is the discrete analogue of the discrepancy principle used by Tautenhahn [15], where the regularization parameter $t_{*}$ is chosen such that

$$
\left\|y^{\delta}-F\left(u\left(t_{*}\right)\right)\right\| \leq \tau \delta<\left\|y^{\delta}-F(u(t))\right\|, \quad 0 \leq t<t_{*} .
$$

Note that the exponential Euler method gives the exact solution for the Showalter differential equation for linear inverse problems. For nonlinear problems this scheme can also be interpreted as Newton's method with asymptotic regularization for solving the linearized problem, see [7] for details. This interpretation allows to apply the convergence results of Rieder [12, 13], which hold for a whole class of Newton methods. However, the convergence rates shown there are not yet optimal.

\section{Convergence analysis}

The aim of this section is to prove the convergence of the exponential Euler regularization for exact and perturbed data. Before we start, we present the analytic framework for our analysis and we review the convergence analysis for the continuous solution of the (Showalter) differential equation (4) from [15].

\subsection{Assumptions}

For the convergence analysis we impose the so-called tangential cone condition, which is also used in $[4,15]$.

Assumption 1. $F: \mathcal{D}(F) \subset X \rightarrow Y$ is Fréchet differentiable and satisfies

$$
\left\|F(\widetilde{x})-F(x)-F^{\prime}(x)(\widetilde{x}-x)\right\| \leq \eta\|F(x)-F(\widetilde{x})\|
$$

for $\eta<0.7$ and for all $x, \widetilde{x} \in B_{r}\left(x_{+}\right) \subset \mathcal{D}(F)$, where $B_{r}\left(x_{+}\right)$denotes the ball of radius $r>0$ around the center $x_{+}$.

This assumption guarantees the following bounds for all $x, \widetilde{x} \in B_{r}\left(x_{+}\right)$

$$
\frac{1}{1+\eta}\left\|F^{\prime}(x)(x-\widetilde{x})\right\| \leq\|F(x)-F(\widetilde{x})\| \leq \frac{1}{1-\eta}\left\|F^{\prime}(x)(x-\widetilde{x})\right\| .
$$

To simplify the presentation we further assume without loss of generality that the problem is appropriately scaled, i.e.,

$$
\left\|F^{\prime}(x)\right\| \leq 1, \quad x \in B_{r}\left(x_{+}\right) .
$$

Remark. Instead of the tangential cone condition, some papers impose that

$$
\left\|F(\widetilde{x})-F(x)-F^{\prime}(x)(\widetilde{x}-x)\right\| \leq C\|x-\widetilde{x}\|\|F(x)-F(\widetilde{x})\|,
$$

for $x, \widetilde{x} \in B_{r}\left(x_{+}\right)$, cf. [4]. Our analysis is valid in this case as well. A discussion about the different conditions can be found in $[5,11]$.

Recall that we always assume that (1) has a solution $x_{*} \in \mathcal{D}(F)$. In this case, Assumption 1 ensures that a unique solution $x_{+}$of minimal distance to $x_{0}$ exists, cf. [11, Prop. 2.1]. This solution satisfies $x_{+}-x_{0} \in \mathcal{N}\left(F^{\prime}\left(x_{+}\right)\right)^{\perp}$. 


\subsection{Continuous case}

We now review the main results on the convergence of the solution of (4). Theorem 3 in [15] states that the method converges when applied to the unperturbed problem (1).

Theorem 3.1. Let Assumption 1 be satisfied and let (1) be solvable in $B_{r}\left(x_{+}\right)$. If $u(t)$ is a solution of (4), then $u(t)$ converges to a solution of $(1)$ in $B_{r}\left(x_{+}\right)$for $t \rightarrow \infty$. If in addition $\mathcal{N}\left(F^{\prime}\left(x_{+}\right)\right) \subset \mathcal{N}\left(F^{\prime}(x)\right)$ for all $x \in B_{r}\left(x_{+}\right)$, then $u(t)$ converges to $x_{+}$.

Theorem 4 of [15] states the corresponding result for perturbed data.

Theorem 3.2. Let Assumption 1, (3), and $\left\|y^{\delta}-F\left(x_{0}\right)\right\|>\tau \delta>0$ be satisfied and let (1) be solvable in $B_{r}\left(x_{+}\right)$. If $u(t)$ is a solution of (4), and $t_{*}$ is chosen from (9) with

$$
\tau>\frac{1+\eta}{1-\eta}
$$

then $u\left(t_{*}\right)$ converges to a solution $x_{*} \in B_{r}\left(x_{+}\right)$of $(1)$ for $\delta \rightarrow 0$. If in addition $\mathcal{N}\left(F^{\prime}\left(x_{+}\right)\right) \subset \mathcal{N}\left(F^{\prime}(x)\right)$ for all $x \in B_{r}\left(x_{+}\right)$, then $u\left(t_{*}\right)$ converges to $x_{+}$for $\delta \rightarrow 0$.

It is shown in [15] that the stopping-time $t_{*}$ is uniquely defined via (9) and finite. An important auxiliary result is the monotonicity of the error function $\left\|u(t)-x_{+}\right\|$. The analogous discrete results will be verified in the analysis of the exponential Euler method below.

\subsection{Discrete case}

Next we analyze the convergence of the exponential Euler method (5). To simplify the presentation of our paper, we introduce the following notation. The operators will be denoted by

$$
\begin{array}{ll}
A_{+}=F^{\prime}\left(x_{+}\right), & A_{n}=F^{\prime}\left(u_{n}\right), \\
J_{+}=A_{+}^{*} A_{+}, & J_{n}=A_{n}^{*} A_{n}, \\
K_{+}=A_{+} A_{+}^{*}, & K_{n}=A_{n} A_{n}^{*},
\end{array}
$$

and the corresponding operator functions by

$$
\begin{array}{ll}
\Phi_{n,+}=\varphi\left(-h_{n} J_{+}\right), & \Phi_{n}=\varphi\left(-h_{n} J_{n}\right), \\
\widetilde{\Phi}_{n,+}=\varphi\left(-h_{n} K_{+}\right), & \widetilde{\Phi}_{n}=\varphi\left(-h_{n} K_{n}\right) .
\end{array}
$$

To ensure monotonicity of the errors (cf. Lemma 3.4 below), the step sizes $h_{n}$ have to be chosen appropriately. For this we propose to use the following discrepancy principle

$$
p_{n}^{\delta}\left(h_{n}\right):=\left\|\psi\left(-h_{n} K_{n}\right) \Delta F_{n}^{\delta}-A_{n} \Delta u_{n}\right\|=\mu\left\|\Delta F_{n}^{\delta}\right\|, \quad \mu<1,
$$

where the updates $\Delta u_{n}$ are written as

$$
\Delta u_{n}=u_{n+1}-u_{n}=h_{n} A_{n}^{*} \widetilde{\Phi}_{n} \Delta F_{n}^{\delta}
$$

and the function $\psi$ is defined by

$$
\psi(z)=\varphi(z)(1-z)
$$

Due to (14), we have

$$
p_{n}^{\delta}\left(h_{n}\right)=\left\|\widetilde{\Phi}_{n} \Delta F_{n}^{\delta}\right\|
$$


We will verify later in Theorems 3.5 and 3.6 that if $h_{n}$ is chosen according to (13), then there exists a parameter $\nu>1$ such that the $n$th residual satisfies

$$
\left\|\psi\left(-h_{n} K_{n}\right) \Delta F_{n}^{\delta}+A_{n} e_{n}\right\| \leq \frac{\mu}{\nu}\left\|\Delta F_{n}^{\delta}\right\|
$$

where

$$
e_{n}=u_{n}-x_{+}
$$

denotes the error of the $n$th iterate $u_{n}$. This will be used to prove existence of a unique step size $h_{n}$ satisfying (13).

Remark. For the Levenberg-Marquardt method the situation simplifies due to $\psi \equiv 1$. In this case, the step size selection (13) and assumption (16) are the same as in $[3]$.

Lemma 3.3. Assume that (16) holds, then there is a unique solution $h_{n}>0$ of (13).

Proof. Obviously, the function $p_{n}^{\delta}(h)$ is continuous and strictly decreasing if $\Delta F_{n}^{\delta} \neq 0$. By $(15)$ and due to $\varphi(0)=1$ and $\varphi(-z) \rightarrow 0$ for $z \rightarrow \infty$ we have

$$
\lim _{h \rightarrow 0} p_{n}^{\delta}(h)=\left\|\Delta F_{n}^{\delta}\right\|, \quad \lim _{h \rightarrow \infty} p_{n}^{\delta}(h)=\left\|\Pi_{n} \Delta F_{n}^{\delta}\right\|,
$$

where $\Pi_{n}$ denotes the orthogonal projection onto $\mathcal{R}\left(A_{n}\right)^{\perp}$. By definition of $\psi$ we have $\psi(0)=1$, and thus

$$
\Pi_{n} \Delta F_{n}^{\delta}=\Pi_{n}\left(\psi\left(-h K_{n}\right) \Delta F_{n}^{\delta}+A_{n}\left(u_{n}-u\right)\right)
$$

for all $u \in \mathcal{D}(F)$. This yields

$$
\left\|\Pi_{n} \Delta F_{n}^{\delta}\right\| \leq\left\|\psi\left(-h K_{n}\right) \Delta F_{n}^{\delta}+A_{n}\left(u_{n}-u\right)\right\|
$$

for all $h>0$ and for all $u \in \mathcal{D}(F)$. The existence of $h_{n}$ satisfying (13) then follows from (16).

The next lemma states that the sequence of errors is monotonically decreasing.

Lemma 3.4. Let $0<\mu<1<\nu$ and assume that (16) is fulfilled so that $h_{n}$ can be defined according to (13). Then for $\Delta u_{n} \neq 0$ we have

$$
\begin{aligned}
& \left\|e_{n}\right\|^{2}-\left\|e_{n+1}\right\|^{2}>\frac{2 h_{n}(\nu-1) \mu}{\nu}\left\|\Delta F_{n}^{\delta}\right\| \cdot\left\|\widetilde{\Phi}_{n} \Delta F_{n}^{\delta}\right\|, \\
& \left\|e_{n}\right\|^{2}-\left\|e_{n+1}\right\|^{2}>-\frac{2 \varphi^{-1}(\mu)(\nu-1) \mu^{2}}{\nu\left\|A_{n}\right\|^{2}}\left\|\Delta F_{n}^{\delta}\right\|^{2} .
\end{aligned}
$$

Note that $\varphi^{-1}(\mu)<0$ for $\mu<1$.

Proof. Our proof is inspired by the proof of Proposition 2.1 in [3]. The errors (17) satisfy

$$
\begin{aligned}
\left\|e_{n+1}\right\|^{2}= & \left\|\Delta u_{n}+e_{n}\right\|^{2} \\
= & \left\|\Delta u_{n}\right\|^{2}+2\left\langle\Delta u_{n}, e_{n}\right\rangle+\left\|e_{n}\right\|^{2} \\
= & h_{n}^{2}\left\langle\Phi_{n} A_{n}^{*} \Delta F_{n}^{\delta}, \Phi_{n} A_{n}^{*} \Delta F_{n}^{\delta}\right\rangle \\
& +2 h_{n}\left\langle\Phi_{n} A_{n}^{*} \Delta F_{n}^{\delta}, e_{n}\right\rangle+\left\|e_{n}\right\|^{2}
\end{aligned}
$$




$$
\begin{aligned}
= & h_{n}^{2}\left\langle\Delta F_{n}^{\delta}, K_{n} \widetilde{\Phi}_{n}^{2} \Delta F_{n}^{\delta}\right\rangle \\
& -2 h_{n}\left\langle\widetilde{\Phi}_{n} \Delta F_{n}^{\delta}, \psi\left(-h_{n} K_{n}\right) \Delta F_{n}^{\delta}\right\rangle \\
& +2 h_{n}\left\langle\widetilde{\Phi}_{n} \Delta F_{n}^{\delta}, \psi\left(-h_{n} K_{n}\right) \Delta F_{n}^{\delta}+A_{n} e_{n}\right\rangle+\left\|e_{n}\right\|^{2} \\
= & -h_{n}^{2}\left\langle\Delta F_{n}^{\delta}, K_{n} \widetilde{\Phi}_{n}^{2} \Delta F_{n}^{\delta}\right\rangle \\
& +2 h_{n}\left\langle\widetilde{\Phi}_{n} \Delta F_{n}^{\delta}, h_{n} K_{n} \widetilde{\Phi}_{n} \Delta F_{n}^{\delta}-\psi\left(-h_{n} K_{n}\right) \Delta F_{n}^{\delta}\right\rangle \\
& +2 h_{n}\left\langle\widetilde{\Phi}_{n} \Delta F_{n}^{\delta}, \psi\left(-h_{n} K_{n}\right) \Delta F_{n}^{\delta}+A_{n} e_{n}\right\rangle+\left\|e_{n}\right\|^{2} .
\end{aligned}
$$

By (14) we have $z \varphi(-z)-\psi(-z)=-\varphi(-z)$, so that the expression above can be written as

$$
\begin{aligned}
\left\|e_{n+1}\right\|^{2}-\left\|e_{n}\right\|^{2}= & -h_{n}^{2}\left\langle\Delta F_{n}^{\delta}, K_{n} \widetilde{\Phi}_{n}^{2} \Delta F_{n}^{\delta}\right\rangle-2 h_{n}\left\langle\widetilde{\Phi}_{n} \Delta F_{n}^{\delta}, \widetilde{\Phi}_{n} \Delta F_{n}^{\delta}\right\rangle \\
& +2 h_{n}\left\langle\widetilde{\Phi}_{n} \Delta F_{n}^{\delta}, \psi\left(-h_{n} K_{n}\right) \Delta F_{n}^{\delta}+A_{n} e_{n}\right\rangle \\
= & -\left\|\Delta u_{n}\right\|^{2}-2 h_{n}\left\|\widetilde{\Phi}_{n} \Delta F_{n}^{\delta}\right\|^{2} \\
& +2 h_{n}\left\langle\widetilde{\Phi}_{n} \Delta F_{n}^{\delta}, \psi\left(-h_{n} K_{n}\right) \Delta F_{n}^{\delta}+A_{n} e_{n}\right\rangle \\
< & -2 h_{n}\left\|\widetilde{\Phi}_{n} \Delta F_{n}^{\delta}\right\|^{2}+2 h_{n}\left\langle\widetilde{\Phi}_{n} \Delta F_{n}^{\delta}, \psi\left(-h_{n} K_{n}\right) \Delta F_{n}^{\delta}+A_{n} e_{n}\right\rangle \\
\leq & 2 h_{n}\left\|\widetilde{\Phi}_{n} \Delta F_{n}^{\delta}\right\|\left(\left\|\psi\left(-h_{n} K_{n}\right) \Delta F_{n}^{\delta}+A_{n} e_{n}\right\|-\left\|\widetilde{\Phi}_{n} \Delta F_{n}^{\delta}\right\|\right) \\
\leq & 2 h_{n} \mu \frac{1-\nu}{\nu}\left\|\widetilde{\Phi}_{n} \Delta F_{n}^{\delta}\right\|\left\|\Delta F_{n}^{\delta}\right\| .
\end{aligned}
$$

For the last inequality we have used (13), (15) and (16).

For the second estimate (19) we note that the relation

$$
\mu\left\|\Delta F_{n}^{\delta}\right\|=\left\|\widetilde{\Phi}_{n} \Delta F_{n}^{\delta}\right\| \geq \varphi\left(-h_{n}\left\|K_{n}\right\|\right)\left\|\Delta F_{n}^{\delta}\right\|
$$

yields

$$
h_{n} \geq-\frac{\varphi^{-1}(\mu)}{\left\|A_{n}\right\|^{2}} .
$$

This proves the desired result.

Using these lemmas, we will prove that the method converges if it is applied to the unperturbed problem (1). We will write the unperturbed problem formally as a perturbed problem with $\delta=0$, so that $y^{\delta}=y$ in our scheme.

Theorem 3.5. Let $0.3+\eta<\mu<1$ and let in addition Assumption 1 and (12) be satisfied. If the step sizes $h_{n}$ are chosen according to (13) and if $y^{\delta}=y=F\left(x_{+}\right)$, then $u_{n}$ converges to a solution of (1) as $n \rightarrow \infty$. If in addition $\mathcal{N}\left(F^{\prime}\left(x_{+}\right)\right) \subset \mathcal{N}\left(F^{\prime}(x)\right)$ for all $x \in B_{r}\left(x_{+}\right)$, then $u_{n}$ converges to $x_{+}$as $n \rightarrow \infty$.

Proof. The proof is done analogously to the proof of Theorem 2.2 in [3]. First we prove that the error decreases monotonically. 
Let $n=0$ and denote by

$$
\Delta F_{n}=y-F\left(u_{n}\right),
$$

the residual of the unperturbed problem. Then, by definition of $\psi$ and (10), we have

$$
\begin{aligned}
\left\|\psi\left(-h_{n} K_{n}\right) \Delta F_{n}+A_{n} e_{n}\right\| & \\
& =\left\|\left(\widetilde{\Phi}_{n}-\exp \left(-h_{n} K_{n}\right)\right) \Delta F_{n}+\Delta F_{n}+A_{n} e_{n}\right\| \\
& \leq\left\|\widetilde{\Phi}_{n}-\exp \left(-h_{n} K_{n}\right)\right\|\left\|\Delta F_{n}\right\|+\left\|\Delta F_{n}+A_{n} e_{n}\right\| \\
& \leq \sup _{z \in[-\infty, 0]}\left|\varphi(z)-\mathrm{e}^{z}\right|\left\|\Delta F_{n}\right\|+\eta\left\|\Delta F_{n}\right\| \\
& \leq(0.3+\eta)\left\|\Delta F_{n}\right\| .
\end{aligned}
$$

Note that the function $\left|\varphi(z)-\mathrm{e}^{z}\right|$ has a unique maximum for $z \leq 0$. The stated bound follows by straightforward numerical calculations. For $\mu>0.3+\eta$ the above reasoning yields (16) with $\nu=\frac{\mu}{0.3+\eta}>1$. Moreover, Lemma 3.4 gives

$$
\left\|e_{n+1}\right\|<\left\|e_{n}\right\| \text {. }
$$

Induction shows that the error decreases monotonically for all $n \in \mathbb{N}$.

As in the proof of Theorem 4.2 of [4], we proceed by proving that $\left\|e_{n}\right\|$ is a Cauchy sequence. Let $m, n \in \mathbb{N}$ be given indices satisfying $m>n$ and let $l$ be an index chosen such that $n \leq l \leq m$ and

$$
\left\|\Delta F_{l}\right\| \leq\left\|\Delta F_{i}\right\|, \quad i=n, \ldots, m .
$$

From (10) we have

$$
\begin{aligned}
\left\|A_{i} e_{l}\right\| & =\left\|A_{i} e_{i}-A_{i}\left(e_{i}-e_{l}\right)\right\| \\
& \leq\left\|\Delta F_{i}+A_{i} e_{i}\right\|+\left\|F\left(u_{i}\right)-F\left(u_{l}\right)-A_{i}\left(e_{i}-e_{l}\right)\right\|+\left\|\Delta F_{l}\right\| \\
& \leq(3 \eta+1)\left\|\Delta F_{i}\right\| .
\end{aligned}
$$

Recall that the error can be written as

$$
e_{n+1}=e_{n}+h_{n} A_{n}^{*} \widetilde{\Phi}_{n} \Delta F_{n} .
$$

Using (18) we obtain

$$
\begin{aligned}
\left|\left\langle e_{l}-e_{n}, e_{l}\right\rangle\right| & =\left|\sum_{i=n}^{l-1}\left\langle h_{i} A_{i}^{*} \widetilde{\Phi}_{i} \Delta F_{i}, e_{l}\right\rangle\right| \\
& =\left|\sum_{i=n}^{l-1}\left\langle h_{i} \widetilde{\Phi}_{i} \Delta F_{i}, A_{i} e_{l}\right\rangle\right| \\
& \leq \frac{(3 \eta+1) \nu}{2(\nu-1) \mu}\left(\left\|e_{n}\right\|^{2}-\left\|e_{l}\right\|^{2}\right) .
\end{aligned}
$$

The identity

$$
\left\|e_{l}-e_{n}\right\|^{2}=2\left\langle e_{l}-e_{n}, e_{l}\right\rangle+\left\|e_{n}\right\|^{2}-\left\|e_{l}\right\|^{2}
$$

thus yields

$$
\left\|e_{l}-e_{n}\right\|^{2} \leq\left(\frac{(3 \eta+1) \nu}{(\nu-1) \mu}+1\right)\left(\left\|e_{n}\right\|^{2}-\left\|e_{l}\right\|^{2}\right)
$$


and analogously

$$
\left\|e_{m}-e_{l}\right\|^{2} \leq\left(\frac{(3 \eta+1) \nu}{(\nu-1) \mu}+1\right)\left(\left\|e_{l}\right\|^{2}-\left\|e_{m}\right\|^{2}\right),
$$

so that

$$
\begin{aligned}
\left\|u_{m}-u_{n}\right\|^{2} & =\left\|e_{m}-e_{n}\right\|^{2} \\
& \leq 2\left\|e_{m}-e_{l}\right\|^{2}+2\left\|e_{l}-e_{n}\right\|^{2} \\
& \leq 2\left(\frac{(3 \eta+1) \nu}{(\nu-1) \mu}+1\right)\left(\left\|e_{n}\right\|^{2}-\left\|e_{m}\right\|^{2}\right) .
\end{aligned}
$$

From the monotonicity of the errors it follows that the sequence $\left\{\left\|e_{n}\right\|\right\}$ converges. Consequently, $\left\{u_{n}\right\}$ is a Cauchy sequence which converges to a limit $u_{\infty}$. By (19) and (12), the series

$$
\sum_{n=0}^{\infty}\left\|\Delta F_{n}\right\|^{2}
$$

converges and therefore $u_{\infty}$ is a solution of (1).

The second part of the theorem, the convergence of $u_{n}$ to $x_{+}$, follows as in Theorem 2.3 in [5].

Our main result of this section is that the exponential Euler iteration applied to the perturbed problem (2) converges to a solution of the unperturbed problem (1) if the perturbation tends to zero.

Theorem 3.6. Let the assumptions of Theorem 3.5 and (3) be satisfied and let $\mu>(1+\eta+\tau(0.3+\eta)) / \tau$. Then the exponential Euler iteration stopped by the discrepancy principle (8) terminates after $n_{*}=n_{*}(\delta)<\infty$ iterations and the corresponding approximations $u_{n_{*}}$ converge to a solution of (1) for $\delta \rightarrow 0$. If in addition $\mathcal{N}\left(F^{\prime}\left(x_{+}\right)\right) \subset \mathcal{N}\left(F^{\prime}(x)\right)$ for all $x \in B_{r}\left(x_{+}\right)$, then $u_{n_{*}}$ converges to $x_{+}$.

Proof. We follow the proof of [3, Theorem 2.3] and show the monotonic decrease of the error as long as the discrepancy principle (8) is not fulfilled. As in the proof of Theorem 3.5 we have for $n \in \mathbb{N}$

$$
\begin{aligned}
& \left\|\psi\left(-h_{n} K_{n}\right) \Delta F_{n}^{\delta}+A_{n} e_{n}\right\| \\
& \leq\left\|\widetilde{\Phi}_{n}-\exp \left(-h_{n} K_{n}\right)\right\|\left\|\Delta F_{n}^{\delta}\right\|+\left\|\Delta F_{n}^{\delta}+A_{n} e_{n}\right\| \\
& \leq \sup _{z \in[-\infty, 0]}\left|\varphi(z)-\mathrm{e}^{z}\right|\left\|\Delta F_{n}^{\delta}\right\|+\delta+\eta\left\|\Delta F_{n}\right\| \\
& \leq(0.3+\eta)\left\|\Delta F_{n}^{\delta}\right\|+\delta(1+\eta) .
\end{aligned}
$$

If $n_{*}=0$, there is nothing to prove. For $n_{*}>0$ and $n<n_{*}$ we have $\delta<\left\|\Delta F_{n}^{\delta}\right\| / \tau$ and hence

$$
\left\|\psi\left(-h_{n} K_{n}\right) \Delta F_{n}^{\delta}+A_{n} e_{n}\right\| \leq \frac{1+\eta+\tau(0.3+\eta)}{\tau}\left\|\Delta F_{n}^{\delta}\right\|,
$$

which shows that (16) holds with $\nu=\mu \tau /(1+\eta+\tau(0.3+\eta))$ for all $n<n_{*}$. Applying Lemma 3.4 finally proves the monotonicity of $\left\{\left\|e_{n}\right\|\right\}$. 
From the discrepancy principle (8), (12), and (19) we conclude

$$
\begin{aligned}
n_{*} \tau^{2} \delta^{2} & \leq \sum_{n=0}^{n_{*}-1}\left\|\Delta F_{n}^{\delta}\right\|^{2} \\
& \leq \frac{\nu}{2 \varphi^{-1}(\mu)(\nu-1) \mu^{2}}\left(\left\|e_{n_{*}}\right\|^{2}-\left\|e_{0}\right\|^{2}\right) \\
& <\infty
\end{aligned}
$$

and therefore the stopping index $n_{*}$ is finite. The remaining part is proved as in [3, Theorem 2.3].

Remark. The convergence results can be generalized by replacing the parameter $\mu$ in (13) by variable parameters $\mu_{n}$ satisfying

$$
\mu_{\min } \leq \mu_{n} \leq \mu_{\max }<1
$$

Theorems 3.5 and 3.6 remain true if the assumptions are fulfilled for $\mu_{\min }$ instead of $\mu$.

\section{Convergence rates}

The aim of this section is to show that the exponential Euler regularization in fact converges with optimal rates under suitable assumptions.

\subsection{Assumptions}

The first additional assumption is a so-called source condition. In many applications, in particular in parameter identification problems for partial differential equations, this condition reflects the smoothness of the initial error.

Assumption 2. There exists $w \in X$ and constants $\gamma \in(0,1 / 2]$ and $\rho \geq 0$ such that

$$
e_{0}=x_{0}-x_{+}=J\left(x_{+}\right)^{\gamma} w, \quad\|w\| \leq \rho
$$

Moreover, we have to assume relations between the Fréchet derivatives $F^{\prime}$ evaluated at certain points in $B_{r}\left(x_{+}\right)$.

Assumption 3. For all $x, \widetilde{x} \in B_{r}\left(x_{+}\right)$there exist linear bounded operators $R(x, \widetilde{x})$ : $Y \rightarrow Y$ and a constant $C_{R} \geq 0$ such that

(i) $F^{\prime}(x)=R(x, \widetilde{x}) F^{\prime}(\widetilde{x})$

(ii) $\|R(x, \widetilde{x})-I\| \leq C_{R}\|x-\widetilde{x}\|$.

Both assumption are standard assumptions arising in the literature, see, e.g. $[10,11]$.

\subsection{Continuous case}

As in Section 3, we first present the main result for the continuous solution of (4) from [15, Theorem 6].

Theorem 4.1. If in addition to the assumptions of Theorem 3.2, Assumptions 2 and 3, and (12) are satisfied and if

$$
\tau>\frac{2-\eta}{1-\eta}
$$


then there exists a constant $c_{*}=c_{*}\left(\tau, \eta, C_{R}, \gamma\right)$ such that

$$
\left\|u\left(t_{*}\right)-x_{+}\right\| \leq c_{*} \rho^{1 /(2 \gamma+1)} \delta^{2 \gamma /(2 \gamma+1)}
$$

for $\rho$ sufficiently small.

This theorem proves that asymptotic regularization converges with optimal rates.

\subsection{Discrete case}

Next we will derive the corresponding results for the discrete exponential Euler regularization scheme. These results are valid under weak restrictions on the step sizes, namely we assume that there exist constants $c_{0}$ and $c_{h}$ such that

$$
h_{0} \leq c_{0}, \quad h_{j} \leq c_{h} t_{j}, \quad j \geq 1,
$$

where, as before, $t_{j+1}=t_{j}+h_{j}$. Note that this step size restriction allows to choose $\left(h_{j}\right)_{j \geq 0}$ as a geometric sequence, i.e. $h_{j}=h_{0} \sigma^{j}$ for some $\sigma>1$.

Our main result is the following theorem.

Theorem 4.2. Let Assumptions 1 to 3 hold and assume that the step sizes $h_{j}$ satisfy (21) for all $j \leq n_{*}$. Here, the stopping index $n_{*}$ is defined by (8), with $\tau$ satisfying (20). Then for $\rho$ sufficiently small, there exists a constant $C=C\left(\tau, \eta, C_{R}, c_{0}, c_{h}, \gamma, r\right)>0$ such that

$$
\left\|e_{n_{*}}\right\| \leq C \rho^{1 /(2 \gamma+1)} \delta^{2 \gamma /(2 \gamma+1)} .
$$

The proof of this theorem is rather involved and therefore split into several pieces. The auxiliary results required will be presented in a series of lemmas. First we give two immediate consequences of our assumptions.

Lemma 4.3. Let Assumption 1 and (3) hold and let the stopping index $n_{*}$ be defined by (8). Then we have

$$
\left\|\Delta F_{n}^{\delta}\right\| \leq \frac{\tau}{(\tau-1)(1-\eta)}\left\|A_{+} e_{n}\right\|, \quad n<n_{*} .
$$

Proof. By (11) and (3) we have

$$
\begin{aligned}
\left\|\Delta F_{n}^{\delta}\right\| & \leq\left\|y^{\delta}-y\right\|+\left\|F\left(x_{+}\right)-F\left(u_{n}\right)\right\| \\
& \leq \delta+\frac{1}{1-\eta}\left\|A_{+} e_{n}\right\| .
\end{aligned}
$$

The estimate thus follows from the stopping criterion (8).

Lemma 4.4. Let Assumption 3 hold. Then, for $x \in B_{r}\left(x_{+}\right)$, we have

$$
\left\|F(x)-F\left(x_{+}\right)-F^{\prime}\left(x_{+}\right)\left(x-x_{+}\right)\right\| \leq \frac{1}{2} C_{R}\left\|x-x_{+}\right\|\left\|F^{\prime}\left(x_{+}\right)\left(x-x_{+}\right)\right\| .
$$

Proof. Proposition 4 in [15].

One of the main ingredients of the theory presented by Tautenhahn [15] is the variation-of-constants formula for the error and the error premultiplied by $A_{+}$. Our analysis uses the discrete variation-of-constants formula (Theorem 4.8), which is derived from the following suitably written error recursion. 
Lemma 4.5. Let Assumption 3 hold. Then the error $e_{n}=u_{n}-x_{+}$of the exponential Euler recursion (5) satisfies

$$
e_{n+1}=\exp \left(-h_{n} J_{+}\right) e_{n}+h_{n} \Phi_{n,+} A_{+}^{*}\left(r_{n}^{(1)}+y^{\delta}-y\right)-h_{n} r_{n}^{(2)} .
$$

where

$$
\begin{aligned}
& r_{n}^{(1)}=F\left(x_{+}\right)-F\left(u_{n}\right)+A_{+} e_{n}+\left(R^{*}\left(u_{n}, x_{+}\right)-I\right) \Delta F_{n}^{\delta}, \\
& r_{n}^{(2)}=\left(\Phi_{n,+}-\Phi_{n}\right) A_{n}^{*} \Delta F_{n}^{\delta} .
\end{aligned}
$$

Proof. By (5), the following error recursion holds

$$
\begin{aligned}
e_{n+1}= & e_{n}+h_{n} \Phi_{n} A_{n}^{*} \Delta F_{n}^{\delta} \\
= & e_{n}+h_{n} \Phi_{n,+} A_{+}^{*}\left(F\left(x_{+}\right)-F\left(u_{n}\right)\right) \\
& -h_{n}\left(\Phi_{n,+} A_{+}^{*}-\Phi_{n} A_{n}^{*}\right) \Delta F_{n}^{\delta} \\
& +h_{n} \Phi_{n,+} A_{+}^{*}\left(y^{\delta}-y\right) .
\end{aligned}
$$

By writing $e_{n}$ in the form

$$
e_{n}=\exp \left(-h_{n} J_{+}\right) e_{n}+h_{n} \Phi_{n,+} A_{+}^{*} A_{+} e_{n}
$$

we get

$$
\begin{aligned}
e_{n+1}= & \exp \left(-h_{n} J_{+}\right) e_{n} \\
& +h_{n} A_{+}^{*} \widetilde{\Phi}_{n,+}\left(F\left(x_{+}\right)-F\left(u_{n}\right)+A_{+} e_{n}\right) \\
& -h_{n}\left(\Phi_{n,+} A_{+}^{*}-\Phi_{n} A_{n}^{*}\right) \Delta F_{n}^{\delta} \\
& +h_{n} A_{+}^{*} \widetilde{\Phi}_{n,+}\left(y^{\delta}-y\right) .
\end{aligned}
$$

The identity

$$
\left.\Phi_{n,+} A_{+}^{*}-\Phi_{n} A_{n}^{*}=\Phi_{n,+} A_{+}^{*}\left(I-R^{*}\left(u_{n}, x_{+}\right)\right)+\left(\Phi_{n,+}-\Phi_{n}\right)\right) A_{n}^{*}
$$

finally proves the desired relation.

Lemma 4.6. Let Assumptions 1 and 3 hold. Then there are constants $C_{i}=$ $C_{i}\left(\tau, \eta, C_{R}, r\right), i=1,2,3$ such that for $j<n \leq n_{*}$ we have

$$
\begin{aligned}
\left\|r_{j}^{(1)}\right\| & \leq C_{1}\left\|e_{j}\right\|\left\|A_{+} e_{j}\right\| \\
\left\|\exp \left(-\left(t_{n}-t_{j+1}\right) J_{+}\right) r_{j}^{(2)}\right\| & \leq C_{2} \frac{1}{\sqrt{1+t_{n}-t_{j}}}\left\|e_{j}\right\|\left\|A_{+} e_{j}\right\| \\
\left\|A_{+} \exp \left(-\left(t_{n}-t_{j+1}\right) J_{+}\right) r_{j}^{(2)}\right\| & \leq C_{3} \frac{1}{1+t_{n}-t_{j}}\left\|e_{j}\right\|\left\|A_{+} e_{j}\right\| .
\end{aligned}
$$

Proof. From Lemmas 4.3 and 4.4 we obtain the first bound with

$$
C_{1}=C_{R}\left(\frac{1}{2}+\frac{\tau}{(\tau-1)(1-\eta)}\right) .
$$

The terms involving $r_{j}^{(2)}$ can be written with the help of the Cauchy integral formula

$$
\Phi_{j,+}-\Phi_{j}=\frac{1}{2 \pi i} \int_{\Gamma} \varphi(\lambda)\left(\left(\lambda I+h_{j} J_{+}\right)^{-1}-\left(\lambda I+h_{j} J_{j}\right)^{-1}\right) \mathrm{d} \lambda,
$$


see [1] and [2]. The contour $\Gamma$ is parameterized by

$$
s \mapsto 1-|s| \mathrm{e}^{i \theta \operatorname{sign} s}, \quad s \in \mathbb{R}
$$

for some $0<\theta<\pi / 2$. Using the resolvent identity

$$
\begin{aligned}
\left(\lambda+h_{j} J_{+}\right)^{-1} & -\left(\lambda+h_{j} J_{j}\right)^{-1} \\
& =h_{j}\left(\lambda+h_{j} J_{+}\right)^{-1}\left(J_{j}-J_{+}\right)\left(\lambda+h_{j} J_{j}\right)^{-1} \\
& =h_{j}\left(\lambda+h_{j} J_{+}\right)^{-1}\left(\left(A_{j}^{*}-A_{+}^{*}\right) A_{j}-A_{+}^{*}\left(A_{+}-A_{j}\right)\right)\left(\lambda+h_{j} J_{j}\right)^{-1} \\
& =h_{j}\left(\lambda+h_{j} J_{+}\right)^{-1} A_{+}^{*}\left(R^{*}\left(u_{j}, x_{+}\right)-R\left(x_{+}, u_{j}\right)\right) A_{j}\left(\lambda+h_{j} J_{j}\right)^{-1}
\end{aligned}
$$

we obtain

$$
\left(\Phi_{j,+}-\Phi_{j}\right) A_{j}^{*}=\frac{1}{2 \pi i} \int_{\Gamma} \varphi(\lambda)\left(\lambda+h_{j} J_{+}\right)^{-1} A_{+}^{*} \Delta R_{j} h_{j} K_{j}\left(\lambda+h_{j} K_{j}\right)^{-1} \mathrm{~d} \lambda
$$

with $\Delta R_{j}=R^{*}\left(u_{j}, x_{+}\right)-R\left(x_{+}, u_{j}\right)$. From

$$
\sup _{\sigma \geq 0}\left|\frac{\sigma}{\lambda+\sigma}\right| \leq 1+\left|\frac{\lambda}{\operatorname{Im} \lambda}\right|=1+\left|\frac{\lambda}{\lambda-1}\right| \frac{1}{\sin \theta}, \quad \lambda \in \Gamma
$$

we have the bound

$$
\left\|\exp \left(-t J_{+}\right) r_{j}^{(2)}\right\| \leq C_{4} \int_{\Gamma}|\varphi(\lambda)|\left\|\exp \left(-t J_{+}\right)\left(\lambda+h_{j} J_{+}\right)^{-1} A_{+}^{*}\right\|\left\|e_{j}\right\| \mathrm{d}|\lambda|\left\|\Delta F_{n}^{\delta}\right\|
$$

with $C_{4}=C_{4}\left(C_{R}, \theta\right)$. By Lemma 4.7, we thus obtain the second bound of Lemma 4.6.

In order to prove the third estimate, we start with

$$
\Phi_{j,+}-\Phi_{j}=\chi_{j,+}-\chi_{j}+\left(I+h_{j} J_{+}\right)^{-1}-\left(I+h_{j} J_{j}\right)^{-1}
$$

with $\chi_{j,+}=\chi\left(-h_{j} J_{+}\right)$and $\chi_{j}=\chi\left(-h_{j} J_{j}\right)$, where

$$
\chi(\lambda)=\varphi(\lambda)-\frac{1}{1-\lambda} .
$$

Note that $\chi(\lambda)=\mathcal{O}\left(\lambda^{-2}\right)$ for $\operatorname{Re} \lambda \rightarrow-\infty$. Using the Cauchy integral formula again yields

$$
\begin{aligned}
& A_{+} \exp \left(-t J_{+}\right)\left(\chi_{j,+}-\chi_{j}\right) A_{j}^{*} \\
& \quad=\frac{1}{2 \pi i} \int_{\Gamma} \chi(\lambda) \exp \left(-t K_{+}\right)\left(\lambda+h_{j} K_{+}\right)^{-1} K_{+} \Delta R_{j} h_{j} K_{j}\left(\lambda+h_{j} K_{j}\right)^{-1} \mathrm{~d} \lambda .
\end{aligned}
$$

By Lemma 4.7, we have

$$
\left\|\exp \left(-t K_{+}\right)\left(\lambda+h_{j} K_{+}\right)^{-1} K_{+}\right\| \leq \frac{C_{5}}{1+t+h_{j}}
$$

which shows that the above integral possesses the desired bound. Using the above resolvent identity with $\lambda=1$ finally shows

$$
\begin{aligned}
& \left.A_{+} \exp \left(-t J_{+}\right)\left(I+h_{j} J_{+}\right)^{-1}-\left(I+h_{j} J_{j}\right)^{-1}\right) A_{j}^{*} \\
& =\exp \left(-t K_{+}\right)\left(I+h_{j} K_{+}\right)^{-1} K_{+} \Delta R_{j} h_{j} K_{j}\left(\lambda+h_{j} K_{j}\right)^{-1} .
\end{aligned}
$$

This can be bounded with the help of Lemma 4.7 . 
Lemma 4.7. For $0 \leq \alpha \leq 1$ and $\lambda \in \Gamma$, where the curve $\Gamma$ is defined by (24), it holds

$$
\begin{aligned}
& \sup _{0 \leq \sigma \leq 1}\left|\sigma^{\alpha} \mathrm{e}^{-\sigma t} \varphi(-\sigma h)\right| \leq \min \left\{\frac{1}{(t+h)^{\alpha}}, \frac{2}{(1+t+h)^{\alpha}}\right\}, \\
& \sup _{0 \leq \sigma \leq 1}\left|\sigma^{\alpha} \mathrm{e}^{-\sigma t} \frac{1}{\lambda+\sigma h}\right| \leq \frac{C_{5}}{(1+t+h)^{\alpha}|\lambda|^{1-\alpha}},
\end{aligned}
$$

with a constant $C_{5}=C_{5}(\theta)$.

Proof. We consider the function $f(x, y)=(1+x+y)^{\alpha} \mathrm{e}^{-x} \varphi(-y)$ on the cone $\Omega=\{x, y \geq 0\}$. It is easily verified that $f$ does not have stationary points in the interior of $\Omega$. Since $f$ is bounded in $\Omega$, local extrema have to appear on the boundary $\partial \Omega$. Obviously, $|f(x, y)| \leq 2$ for $(x, y) \in \partial \Omega$. The other bounds follows in the same way.

We are now ready to prove that the errors $\left\|e_{n}\right\|$ and $\left\|A_{+} e_{n}\right\|$ decay with the same rate proportional to $\left(1+t_{n}\right)^{\gamma}$ and $\left(1+t_{n}\right)^{\gamma+1 / 2}$, respectively, as their continuous counterparts in [15].

Theorem 4.8. Let the assumptions of Theorem 4.2 hold. Then for $\rho$ sufficiently small there is a constant $C_{*}=C_{*}\left(\tau, \eta, C_{R}, c_{0}, c_{h}, \gamma, r\right)$ such that for $n<n_{*}$

$$
\begin{aligned}
\left\|e_{n}\right\| & \leq C_{*} \frac{\rho}{\left(1+t_{n}\right)^{\gamma}}, \\
\left\|A_{+} e_{n}\right\| & \leq C_{*} \frac{\rho}{\left(1+t_{n}\right)^{\gamma+1 / 2}} .
\end{aligned}
$$

Proof. For an arbitrary $n \in \mathbb{N}$ the error recursion (22) leads to the following discrete variation-of-constants formulas

$$
\begin{aligned}
e_{n} & =\exp \left(-t_{n} J_{+}\right) e_{0} \\
& +\sum_{j=0}^{n-1} h_{j} \exp \left(-\left(t_{n}-t_{j+1}\right) J_{+}\right)\left(\Phi_{j,+} A_{+}^{*}\left(r_{j}^{(1)}+y^{\delta}-y\right)-r_{j}^{(2)}\right)
\end{aligned}
$$

and

$$
\begin{aligned}
A_{+} e_{n} & =\exp \left(-t_{n} K_{+}\right) A_{+} e_{0} \\
& +\sum_{j=0}^{n-1} h_{j} \exp \left(-\left(t_{n}-t_{j+1}\right) K_{+}\right) \widetilde{\Phi}_{j,+} K_{+}\left(r_{j}^{(1)}+y^{\delta}-y\right) \\
& -\sum_{j=0}^{n-1} h_{j} A_{+} \exp \left(-\left(t_{n}-t_{j+1}\right) J_{+}\right) r_{j}^{(2)} .
\end{aligned}
$$

By Lemma 4.7, the sum multiplying $y^{\delta}-y$ in (26) can be bounded by

$$
\begin{aligned}
\left\|\sum_{j=0}^{n-1} h_{j} \exp \left(-\left(t_{n}-t_{j+1}\right) J_{+}\right) A_{+}^{*} \widetilde{\Phi}_{j,+}\right\| & \leq \sum_{j=0}^{n-1} \frac{h_{j}}{\sqrt{t_{n}-t_{j}}} \\
& \leq \int_{0}^{t_{n}} \frac{1}{\sqrt{t_{n}-s}} \mathrm{~d} s=2 \sqrt{t_{n}}
\end{aligned}
$$


while the corresponding sum in (27) can be bounded by one by using the (telescopic) identity

$$
\sum_{j=0}^{n-1} h_{j} \exp \left(-\left(t_{n}-t_{j+1}\right) K_{+}\right) K_{+} \widetilde{\Phi}_{j,+}=I-\exp \left(-t_{n} K_{+}\right) .
$$

Thus, by Assumption 2, Lemmas 4.6 and 4.7 we have

$$
\begin{aligned}
\left\|e_{n}\right\| \leq & \frac{\rho}{\left(1+t_{n}\right)^{\gamma}}+2 \sqrt{t_{n}} \delta \\
& +\left(2 C_{1}+C_{2}\right) \sum_{j=0}^{n-1} h_{j} \frac{1}{\sqrt{1+t_{n}-t_{j}}}\left\|e_{j}\right\|\left\|A_{+} e_{j}\right\|
\end{aligned}
$$

and

$$
\begin{aligned}
\left\|A_{+} e_{n}\right\| \leq & \frac{\rho}{\left(1+t_{n}\right)^{\gamma+1 / 2}}+\delta \\
& +\left(2 C_{1}+C_{3}\right) \sum_{j=0}^{n-1} h_{j} \frac{1}{1+t_{n}-t_{j}}\left\|e_{j}\right\|\left\|A_{+} e_{j}\right\| .
\end{aligned}
$$

The proof now proceeds by induction for $n=0,1, \ldots, n_{*}-1$. By Assumption 2, the statement is true for $n=0$ if $C_{*} \geq 1$. Assuming that the bounds hold for all indices up to $n-1$, we obtain

$$
\left\|e_{n}\right\| \leq \frac{\rho}{\left(1+t_{n}\right)^{\gamma}}+2 \sqrt{t_{n}} \delta+C_{*}^{2} \rho^{2}\left(2 C_{1}+C_{2}\right) S_{n}\left(\frac{1}{2}, 2 \gamma+\frac{1}{2}\right)
$$

and

$$
\left\|A_{+} e_{n}\right\| \leq \frac{\rho}{\left(1+t_{n}\right)^{\gamma+1 / 2}}+\delta+C_{*}^{2} \rho^{2}\left(2 C_{1}+C_{3}\right) S_{n}\left(1,2 \gamma+\frac{1}{2}\right)
$$

where

$$
S_{n}(\alpha, \beta)=\sum_{j=0}^{n-1} \frac{h_{j}}{\left(1+t_{n}-t_{j}\right)^{\alpha}\left(1+t_{j}\right)^{\beta}} .
$$

The inequalities in Lemma 4.11 below lead to

$$
\begin{aligned}
\left\|e_{n}\right\| & \leq \frac{\rho}{\left(1+t_{n}\right)^{\gamma}}\left(1+C_{*}^{2} \rho C_{6}\right)+2 \sqrt{t_{n}} \delta, \\
\left\|A_{+} e_{n}\right\| & \leq \frac{\rho}{\left(1+t_{n}\right)^{\gamma+1 / 2}}\left(1+C_{*}^{2} \rho C_{7}\right)+\delta
\end{aligned}
$$

with

$$
\begin{aligned}
& C_{6}=\left(2 C_{1}+C_{2}\right) C_{9}, \\
& C_{7}=\left(2 C_{1}+C_{3}\right) C_{9} .
\end{aligned}
$$

Applying (8) and Lemma 4.3 we get

$$
\begin{aligned}
\delta & \leq \frac{1}{(\tau-1)(1-\eta)}\left\|A_{+} e_{n}\right\| \\
& \leq \frac{1}{(\tau-1)(1-\eta)}\left(\frac{\rho}{\left(1+t_{n}\right)^{\gamma+1 / 2}}\left(1+C_{*}^{2} \rho C_{7}\right)+\delta\right) .
\end{aligned}
$$


By (20), this yields for $n<n_{*}$

$$
\delta \leq C_{8} \frac{\rho}{\left(1+t_{n}\right)^{\gamma+1 / 2}}
$$

with

$$
C_{8}=\frac{1}{(\tau-1)(1-\eta)-1}\left(1+C_{*}^{2} \rho C_{7}\right) .
$$

Inserting this relation into (31) shows

$$
\left\|e_{n}\right\| \leq \frac{\rho}{\left(1+t_{n}\right)^{\gamma}}\left(1+C_{*}^{2} \rho C_{6}+2 C_{8}\right) .
$$

This yields the desired result, as long as

$$
\begin{aligned}
& 1+C_{*}^{2} \rho C_{6}+2 C_{8} \leq C_{*}, \\
& 1+C_{*}^{2} \rho C_{7}+C_{8} \leq C_{*}
\end{aligned}
$$

hold. This can be achieved for $\rho$ sufficiently small.

Remark. If the maximum possible step sizes $h_{j}=c_{h} t_{j}, j=1, \ldots, n_{*}-1$ are chosen, then Lemma 4.3, Theorem 4.8, and (8) show that there is a constant $c$ such that the stopping index satisfies $n_{*} \leq c|\log \delta|$.

Theorem 4.9. Let the assumptions of Theorem 4.2 be satisfied. If in addition the step size sequence is bounded away from 0, then the stopping index $n_{*}$ defined by (8) is finite and the iterates $u_{n}$ satisfy $u_{n} \in B_{C_{*} \rho}\left(x_{+}\right)$for $n=0,1, \ldots, n_{*}$, and $C_{*}$ as in Theorem 4.8. Moreover, the approximation $u_{n_{*}}$ converges to a solution of (1) for $\delta \rightarrow 0$.

Proof. The assumption that the step sizes are bounded away from 0 ensures that $t_{n} \rightarrow \infty$ for $n \rightarrow \infty$. By Lemma 4.3 and Theorem 4.8 we have that $\left\|\Delta F_{n}^{\delta}\right\| \rightarrow 0$ for $n \rightarrow \infty$. Hence the discrepancy principle (8) is satisfied for $n_{*}<\infty$. Moreover, by Theorem 4.8 we have $\left\|e_{n}\right\| \leq C_{*} \rho$ for $n=0,1, \ldots$, which shows that the method is well-defined with $u_{n} \in B_{C_{*} \rho}\left(x_{+}\right)$.

We close this section with two auxiliary lemmas which are used to prove our main theorem.

Lemma 4.10. Let $f_{T}:[0, T] \rightarrow[0, \infty), T>0$, be a family of convex integrable functions and let $I:[0, \infty) \rightarrow[0, \infty)$ be a function such that

$$
f_{T}(0) \leq C_{f} I(T) \quad \text { and } \quad \int_{0}^{T} f_{T}(t) \mathrm{d} t \leq I(T) .
$$

Then, for any step size sequence $\left(h_{j}\right)_{0 \leq j \leq n-1}$ satisfying (21), we have

$$
\sum_{j=0}^{n-1} h_{j} f_{t_{n}}\left(t_{j}\right) \leq C_{I} I\left(t_{n}\right), \quad C_{I}=1+c_{0} C_{f}+c_{h} .
$$

We stress the fact that the constant $C_{I}$ is independent of the particular choice of the step size sequence. 


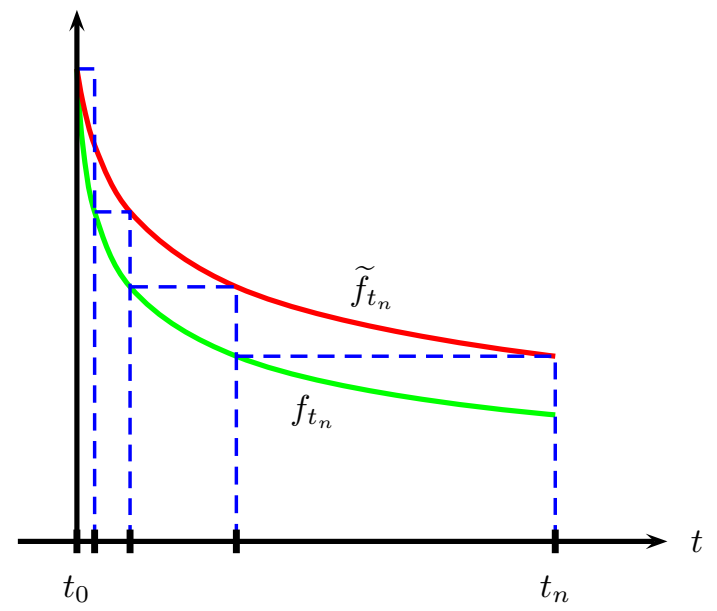

Figure 1. Illustration of the construction in the proof of Lemma 4.10.

Proof. Let $T^{*}$ denote the point in $\left[0, t_{n}\right]$ at which $f_{t_{n}}$ achieves its minimum. Then $f_{t_{n}}$ is monotonically decreasing on $\left[0, T^{*}\right]$. For $0 \leq t \leq\left(1+c_{h}\right) T^{*}$ we define the auxiliary function

$$
\tilde{f}_{t_{n}}(t)=f_{t_{n}}\left(\frac{1}{1+c_{h}} t\right)
$$

This function is constructed such that on the interval $\left[0, T^{*}\right]$, any Riemann sum of $f_{t_{n}}$ is a lower sum if the step size sequence satisfies (21), see Fig. 1 for an illustration. Let $1 \leq j^{*} \leq n$ be such that $t_{j^{*}-1}<T^{*} \leq t_{j^{*}}$. By construction we have

$$
\begin{aligned}
\int_{0}^{T^{*}} f_{t_{n}}(t) \mathrm{d} t & \leq \sum_{j=0}^{j^{*}-1} h_{j} f_{t_{n}}\left(t_{j}\right) \\
& \leq h_{0} f_{t_{n}}(0)+\int_{0}^{\left(1+c_{h}\right) T^{*}} \widetilde{f}_{t_{n}}(t) \mathrm{d} t \\
& =h_{0} f_{t_{n}}(0)+\left(1+c_{h}\right) \int_{0}^{T^{*}} f_{t_{n}}(t) \mathrm{d} t .
\end{aligned}
$$

For $t \geq T^{*}$, the Riemann sum is in fact a lower sum for the integral so that

$$
\sum_{j=j^{*}}^{n-1} h_{j} f_{t_{n}}\left(t_{j}\right) \leq \int_{T^{*}}^{t_{n}} f_{t_{n}}(t) \mathrm{d} t .
$$

Due to the assumptions this finally yields (34).

Lemma 4.11. Let $0<\gamma \leq 1 / 2$ and let $S_{n}$ be given by (30) and assume that (21) holds. Then, for $\alpha=\frac{1}{2}, 1, \frac{1}{2}-\gamma$, there is a constant $C_{9}=C_{9}\left(\alpha, \gamma, c_{0}, c_{h}\right)$ such that

$$
S_{n}\left(\alpha, 2 \gamma+\frac{1}{2}\right) \leq C_{9} \frac{1}{\left(1+t_{n}\right)^{\alpha+\gamma-1 / 2}} .
$$


Proof. The bound follows from Lemma 4.10 for the function

$$
f_{t_{n}}=\left(1+t_{n}-t\right)^{-\alpha}(1+t)^{-\beta}, \quad \beta=2 \gamma+\frac{1}{2} .
$$

This function satisfies all the assumptions of Lemma 4.10 with

$$
I(t)=I\left(t, \alpha, 2 \gamma+\frac{1}{2}\right)=c_{\alpha, \gamma} \frac{1}{(1+t)^{\alpha+\gamma-1 / 2}}, \quad \alpha=\frac{1}{2}, 1, \frac{1}{2}-\gamma,
$$

for some constants $c_{\alpha, \gamma}$ and $C_{f}=1 / c_{\alpha, \gamma}$, see [15, Proposition 6].

It remains to prove our main theorem.

Proof of Theorem 4.2. We write (26) for $n=n_{*}$ in the form

$$
e_{n_{*}}=J_{+}^{\gamma} v_{*}+\sum_{j=0}^{n_{*}-1} h_{j} \exp \left(-\left(t_{n_{*}}-t_{j+1}\right) J_{+}\right) \Phi_{j,+} A_{+}^{*}\left(y^{\delta}-y\right)
$$

where

$$
v_{*}=\exp \left(-t_{n_{*}} J_{+}\right) w+\sum_{j=0}^{n_{*}-1} h_{j} \exp \left(-\left(t_{n_{*}}-t_{j+1}\right) J_{+}\right) J_{+}^{-\gamma}\left(\Phi_{j,+} A_{+}^{*} r_{j}^{(1)}-r_{j}^{(2)}\right) .
$$

Note that $v_{*}$ is well defined as $r_{j}^{(2)} \in \mathcal{R}\left(A_{+}^{*}\right)$, see (25), and since

$$
J_{+}^{-\gamma} A_{+}^{*}: \mathcal{N}\left(A_{+}^{*}\right)^{\perp} \rightarrow X
$$

is a bounded operator for $\gamma \leq \frac{1}{2}$.

Using (25) and Lemmas 4.7 and 4.11 , we bound $v_{*}$ by

$$
\begin{aligned}
\left\|v_{*}\right\| & \leq \rho+C_{*}^{2} \rho^{2}\left(2 C_{1}+C_{5}\right) S_{n_{*}}\left(\frac{1}{2}-\gamma, 2 \gamma+\frac{1}{2}\right) \\
& \leq C_{10} \rho
\end{aligned}
$$

with

$$
C_{10}=1+C_{*}^{2} \rho\left(2 C_{1}+C_{5}\right) C_{9} .
$$

Next using the telescopic identity (29)

$$
\begin{aligned}
A_{+} J_{+}^{\gamma} v_{*} & =A_{+} e_{n_{*}}-\sum_{j=0}^{n_{*}-1} h_{j} \exp \left(-\left(t_{n_{*}}-t_{j+1}\right) K_{+}\right) K_{+} \widetilde{\Phi}_{j,+}\left(y^{\delta}-y\right) \\
& =A_{+} e_{n_{*}}+\left(\exp \left(-t_{n_{*}} K_{+}\right)-I\right)\left(y^{\delta}-y\right)
\end{aligned}
$$

implies

$$
\begin{aligned}
\left\|J_{+}^{\gamma+1 / 2} v_{*}\right\|=\left\|A_{+} J_{+}^{\gamma} v_{*}\right\| & \leq\left\|A_{+} e_{n_{*}}\right\|+\delta \\
& \leq(1+\eta)\left\|F\left(u_{n_{*}}\right)-F\left(x_{+}\right)\right\|+\delta \\
& \leq(1+\eta)\left(\left\|\Delta F_{n_{*}}^{\delta}\right\|+\delta\right)+\delta \\
& \leq C_{11} \delta
\end{aligned}
$$

with

$$
C_{11}=(1+\eta)(1+\tau)+1
$$


by (11) and the discrepancy principle (8).

With the help of the moment inequality, we obtain

$$
\begin{aligned}
\left\|J_{+}^{\gamma} v_{*}\right\| & \leq\left\|J_{+}^{\gamma+1 / 2} v_{*}\right\|^{2 \gamma /(2 \gamma+1)}\left\|v_{*}\right\|^{1 /(2 \gamma+1)} \\
& \leq\left(C_{11} \delta\right)^{2 \gamma /(2 \gamma+1)}\left(C_{10} \rho\right)^{1 /(2 \gamma+1)}
\end{aligned}
$$

Using (28), we obtain

$$
\left\|e_{n_{*}}\right\| \leq\left\|J_{+}^{\gamma} v_{*}\right\|+2 \sqrt{1+t_{n_{*}}} \delta .
$$

By (33) for $n=n_{*}-1$ we further have

$$
\delta \leq C_{8}\left(1+c_{h}\right)^{\gamma+1 / 2} \frac{\rho}{\left(1+t_{n_{*}}\right)^{\gamma+1 / 2}}
$$

so that

$$
\sqrt{1+t_{n_{*}}} \delta^{1 /(2 \gamma+1)} \leq C_{8}^{1 /(2 \gamma+1)}\left(1+c_{h}\right)^{1 / 2} \rho^{1 /(2 \gamma+1)} .
$$

Inserting this into (36) and defining

$$
C=C_{11}^{2 \gamma /(2 \gamma+1)} C_{10}^{1 /(2 \gamma+1)}+2 C_{8}^{1 /(2 \gamma+1)}\left(1+c_{h}\right)^{1 / 2}
$$

gives the desired result.

\section{Concluding Remarks}

In this paper we proved that the exponential Euler regularization method converges with optimal rates under suitable assumptions. If the step sizes are chosen according to the discrepancy principle (13), then the method converges without requiring a source condition. If the source condition (cf. Assumption 2) is satisfied, then Theorem 4.2 shows that the rate of convergence is optimal, if the step sizes chosen by (13) do not grow faster than (21). Note that (21) is satisfied if $h_{j+1} / h_{j} \leq$ const, $j=0,1, \ldots$, so that this result appears to be relevant for practical applications. However, if (21) fails to be true, then the results of Theorems 4.2 and 4.9 guarantee that one can switch to any step size sequence satisfying $h_{\min } \leq h_{j} \leq c_{h} t_{j}$ and still gets optimal convergence rates.

It is possible to generalize the results of this paper to other methods, for instance to the closely related Levenberg-Marquardt scheme, where the function $\varphi$ in (5) is taken as

$$
\varphi(z)=(1-z)^{-1}
$$

As noted before, Hanke [3] proved that the convergence results of Section 3 hold without any changes for this method as well. A proof of optimal convergence rates is presented in [6]. The convergence of more general methods will be studied elsewhere.

Further, we would like to mention the recent paper by Jin and Tautenhahn [10], where the authors analyze Newton type methods of the form

$$
u_{n+1}=x_{0}+h_{n} \varphi\left(-h_{n} J_{n}\right) A_{n}^{*}\left(\Delta F_{n}^{\delta}+A_{n}\left(u_{n}-x_{0}\right)\right) .
$$

In that paper, optimal convergence rates have been proved for a whole class of $\varphi$ functions, among those the function (7) of the exponential Euler scheme. The techniques used in that paper, however, are quite different from ours and cannot be used to analyze our method (5). 
During the revision of the present paper, the new preprint [9] by Jin appeared where optimal convergence rates for the Levenberg-Marquardt scheme for an a priori given step size sequence $h_{j}=h_{0} \sigma^{j}, j \geq 0$ are shown.

\section{Acknowledgement}

We would like to thank Martin Hanke for his careful reading of a preliminary version of this manuscript and for helpful discussions. We further thank the anonymous referee for constructive criticism which enforced us to work harder on some of our estimates so that we could finally considerably weaken our conditions on the step sizes.

\section{References}

[1] A.B. Bakushinsky and M.Yu. Kokurin. Iterative Methods for Approximate Solution of Inverse Problems. Springer, Dordrecht, 2004.

[2] N. Dunford and J.T. Schwartz. Linear Operators. Part I: General Theory. Reprint of the 1958 original. John Wiley \& Sons, New York, 1988.

[3] M. Hanke. A regularizing Levenberg-Marquardt scheme, with applications to inverse groundwater filtration problems. Inverse Problems, 13(1):79-95, 1997.

[4] M. Hanke. Regularizing properties of a truncated Newton-cg algorithm for nonlinear inverse problems. Numer. Funct. Anal. Optim., 18(9 \& 10):971-993, 1997.

[5] M. Hanke, A. Neubauer, and O. Scherzer. A convergence analysis of the Landweber iteration for nonlinear ill-posed problems. Numerische Mathematik, 72(1):21-37, 1995.

[6] M. Hochbruck and M. Hönig. On the convergence of a regularizing Levenberg-Marquardt scheme for nonlinear ill-posed problems. Technical report, Mathematisches Institut, Heinrich-HeineUniversität Düsseldorf, Germany, 2009.

[7] M. Hochbruck, M. Hönig, and A. Ostermann. Regularization of nonlinear ill-posed problems by exponential integrators. Mathematical Modelling and Numerical Analysis, to appear.

[8] M. Hochbruck, A. Ostermann, and J. Schweitzer. Exponential Rosenbrock-type methods. SIAM J. Numer. Anal., 47(1):786-803, 2009.

[9] Q. Jin. On a regularized Levenberg-Marquardt method for solving nonlinear inverse problems. Technical report, University of Texas at Austin, 2009.

[10] Q. Jin and U. Tautenhahn. On the discrepancy principles for some Newton type methods for solving nonlinear inverse problems. Numerische Mathematik, 111(4):509-558, 2009.

[11] B. Kaltenbacher, A. Neubauer, and O. Scherzer. Iterative Regularization Methods for Nonlinear Ill-Posed Problems. De Gruyter, Berlin, New York, 2008.

[12] A. Rieder. On the regularization of nonlinear ill-posed problems via inexact Newton iterations. Inverse Problems, 15(1):309-327, 1999.

[13] A. Rieder. On convergence rates of inexact Newton regularizations. Numerische Mathematik, 88(2):347-365, 2001.

[14] D. Showalter. Representation and computation of the pseudoinverse. Proceedings of the American Mathematical Society, 18:584-586, 1967.

[15] U. Tautenhahn. On the asymptotical regularization of nonlinear ill-posed problems. Inverse Problems, 10(6):1405-1418, 1994. 